Psychological Medicine, 1998, 28, 253-255. Printed in the United Kingdom

(C) 1998 Cambridge University Press

\title{
EDITORIAL
}

\section{Back to the future: the neurobiology of major depression ${ }^{1}$}

This issue of Psychological Medicine carries a number of papers on the neurobiology of major depression. Those acquainted with the field will recognize some familiar topics and while familiarity can be comforting, it can also raise a sense of unease; as the millennium approaches should we still be enquiring about the role of decreased serotonin and increased cortisol in the pathophysiology of depressive disorders?

In our defence we can maintain that there is, in fact good evidence that serotonin and cortisol are implicated in the biology of depression. Our failure to tie down exactly how they are involved in the manifestation of the depressive syndrome does not reflect their lack of importance but rather the problems we have in asking the right questions and the technical difficulties we have answering them. So how far do the papers in this issue take us into this difficult territory?

\section{CORTISOL, DHEA AND COGNITION}

Cortisol is known to be a stress-responsive hormone that can have prominent effects on mood. It is, therefore, a plausible biological mediator through which the effects of psychosocial stress can be translated into clinical mood disorders in vulnerable individuals (Herbert, 1997). Despite this popular hypothesis we do not know whether patients vulnerable to depression have altered cortisol responses to psychosocial stress. In addition, there is little prospective data on the prognostic value of persistent cortisol elevations although there is some agreement that in patients with major depression continued failure to suppress cortisol in response to dexamethasone predicts a relatively poor clinical outcome (Ribeiro et al. 1993).

The paper by Goodyer et al. (1998) shows two important developments in this area. The first, is the recognition that adrenal steroids other than cortisol are likely to be important in mediating the effect of the hypothalamo-pituitary-adrenal (HPA) axis on the central nervous system. Accordingly, these authors have measured concentrations of dehydroepiandosterone (DHEA) as well as cortisol. DHEA has aroused much interest in recent years. Its concentration in plasma is about ten times that of cortisol but unlike cortisol, peak levels of DHEA over the lifespan occur at about the age of 20 years with a steady decline thereafter. There is great interest in whether this decrease in plasma DHEA may play a role predisposing to a wide range of age-related disorders (Herbert, 1995).

A key property of DHEA for the present argument is that it appears to possess antiglucorticoid activity; it may, therefore, offset some of the deleterious cellular effects of corticosteroids (Blauer et al. 1991). If this is the case, the ratio of cortisol to DHEA may provide a better measure of HPA axis activation than either steroid considered alone. In fact, it appears that in the child and adolescent patients studied by Goodyer et al. higher evening cortisol/DHEA ratios did predict persistent depression, while cortisol concentrations alone did not. If these results can be replicated they would suggest that measurements of DHEA should be added to those of cortisol in the assessment of HPA axis activity in depression.

Another development in recent studies, again demonstrated by this paper, is the use of the sophisticated measuring instruments of social psychiatry to establish how social adversity may relate to HPA axis abnormalities (and vice versa). In this paper the presence of 'disappointing' life events also increased the risk of persistent depression and, intriguingly, subjects with high

\footnotetext{
${ }^{1}$ Address for correspondence: Professorr P. J. Cowen, University Department of Psychiatry, Warneford Hospital, Oxford OX3 7JX.
} 
cortisol/DHEA ratios were more likely to experience such life events. This leads the authors to speculate that one of the deleterious neurobiological effects of hypercortisolaemia could be to impair aspects of social cognition, thereby increasing the risk that such life events may be experienced.

There is, in fact, growing interest in the effect of cortisol on cognition, a topic addressed by Van Londen et al. (1998) in their study of depressed patients. There is a good body of evidence, from both human and animal studies, that excessive cortisol secretion can impair learning and memory (Sapolsky et al. 1986; Kirschbaum et al. 1996). Conceivably then, excessive cortisol secretion could account for the cognitive deficits seen in depressed patients. While the literature is not without inconsistency the data of Van Londen et al. support those studies in which cortisol concentrations have been found to relate inversely to cognitive performance in depressed patients.

Against all this we need to keep the perspective that increased cortisol secretion is a normal and presumably adaptive response to stress. Are there positive 'trade-offs', on cognitive performance and on mood, that we need to establish? On the other hand, is it possible that subjects vulnerable to mood disturbance react in a different way to increased cortisol secretion? Whether or not cortisol produces different neurobiological effects in those vulnerable to depression should be a tractable question.

\section{TRYPTOPHAN DEPLETION, 5-HT AND MOOD}

The paper by Neumeister et al. (1998) uses a technique called 'tryptophan depletion' that has attracted growing interest since its effects on mood in healthy subjects were first described by Young et al. (1985). The synthesis of 5-HT in the brain is dependent on the availability of its amino acid precursor, tryptophan (TRP), from plasma and manipulations that decrease plasma TRP concentrations can lower brain 5-HT synthesis (Fernstrom \& Wurtman, 1971).

In TRP depletion subjects are given a mixture of amino acids from which TRP has been omitted. Ingestion and absorption of this mixture stimulates protein synthesis, a process requiring TRP which is an essential amino acid. Because TRP has been omitted from the mixture the body uses its own tissue stores of TRP to manufacture protein (Gessa et al. 1974). This causes plasma TRP concentrations to drop to about $20 \%$ of normal values over the next $5 \mathrm{~h}$. In addition, the neutral amino acids in the mixture compete with TRP for active transport across the blood-brain barrier, further lowering brain availability of TRP. Recent investigations using positron emission tomography (PET) with $\alpha-\left[11^{\mathrm{C}}\right]$ methyl-L-tryptophan have confirmed that TRP depletion lowers brain 5-HT synthesis in humans (Nishizawa et al. 1997).

TRP depletion provides a simple dietary means of lowering brain 5-HT synthesis. It, therefore, allows direct investigation as to whether lowering brain 5-HT function does indeed play an important role in the pathophysiology of mood disorders. In its simplest form the 5-HT hypothesis of depression would predict that lowering brain 5-HT neurotransmission would be sufficient to cause clinical depression. This is clearly not the case. In subjects with no personal or family history of depression TRP depletion may cause a mild and inconsistent lowering of mood (Young et al. 1985; Smith et al. 1997a) but clinical symptomatology is not seen.

The paper by Neumeister $e t$ al. adds to a growing body of data suggesting that in subjects with a history of depression (both seasonal and non-seasonal) who are fully recovered and off drug treatment, TRP depletion can cause an acute, temporary, symptomatic relapse (Moreno et al. 1996; Smith et al. 1997b). This suggests that low 5-HT may indeed play a role in the pathophysiology of depression but that it must interact with some other factor to produce clinical symptomatology.

It is conceivable that this factor could reside in the regulation of 5-HT synthesis. Perhaps those with a vulnerability to depression experience a greater impairment in 5-HT neurotransmission when TRP levels are lowered. PET imaging may provide a means of answering this question. It is, however, also quite possible that those vulnerable to depression have an abnormality in specific groups of neurones crucial to mood regulation and that neurotransmitters like 5-HT act to repair or protect these neurones perhaps by increasing intracellular levels of neurotropic factors (Duman 
et al. 1997). From this viewpoint, lowering brain 5-HT neurotransmission could allow a critical underlying abnormality to be revealed.

Clearly, lowering brain 5-HT function by TRP depletion is a highly artificial manoeuvre. If diminished brain 5-HT function can precipitate depression in vulnerable subjects how might this occur in real life? Interestingly, there may be a role for HPA axis activation because animal studies suggest that excessive cortisol secretion can lower brain 5-HT function by a number of mechanisms including stimulation of TRP metabolism and decreased expression of certain 5-HT receptors (Maes et al. 1990; Chaouloff, 1995).

These effects of cortisol give rise to some intriguing possibilities by which psychosocial stress, HPA axis activation and lowered brain 5-HT function could interact to cause depression and cognitive impairment in vulnerable individuals (Deakin \& Graeff, 1991). There are also interesting questions about the impact of other risk factors such as genetic influences, early life experiences and temperament on these social and biological mechanisms. Our task is difficult because we have to ask questions that on the one hand permit the testing of clear and critical hypotheses and on the other do justice to the complexity of the biosocial mechanisms that we are studying. Unfortunately, biological psychiatry has not always been able to avoid the problem identified by Dr Johnson in one of his colleagues - 'that fellow seems to me to possess but one idea, and that is a wrong one'.

P. J. COWEN

\section{REFERENCES}

Blauer, K. I., Poth, M., Rogers, W. M. \& Bernton, E. W. (1991) Dehydroepiandosterone antagonises the suppressive effect of dexamethasone on lymphocyte proliferation. Endocrinology 129, 3174-3179.

Chaouloff, F. (1995). Regulation of 5-HT receptors by corticosteroids: where do we stand? Fundamental Clinical Pharmacology 9, 219-233.

Deakin, J. F. W. \& Graeff, F. G. (1991). 5-HT and mechanisms of defence. Journal of Psychopharmacology 5, 305-315.

Duman, R. S., Heninger, G. R. \& Nestler, E. J. (1997). A molecular and cellular theory of depression. Archives of General Psychiatry 54, 597-606.

Fernstrom, J. D. \& Wurtman, R. J. (1971). Brain serotonin content: physiological dependence on plasma tryptophan levels. Science 173, 149-152.

Gessa, G. L., Biggio, G., Fadda, F., Corsini, G. V. \& Tagliamonte, A. (1974). Effect of the oral administration of tryptophan-free amino acid mixtures on serum tryptophan, brain tryptophan and serotonin metabolism. Journal of Neurochemistry 22, 869-870

Goodyer, I. M., Herbert, J. \& Althan, P. M. E. (1998). Adrenal steroid secretion and major depression in 8- to 16-year-olds, III. Influence of cortisol/DHEA ratio at presentation on subsequent rates of disappointing life events and persistent major depression. Psychological Medicine 28, 265-273.

Herbert, J. (1995). The age of dehydroapiandosterone. Lancet 345, 1193-1194.

Herbert, J. (1997). Stress, the brain and mental illness. British Medical Journal 315, 530-535.

Kirschbaum, C., Wolf, O. T., May, M., Wippich, W. \& Hellhammer, D. H. (1996). Stress- and treatment-induced elevation of cortisol levels associated with impaired declarative memory in healthy adults. Life Sciences 58, 1475-1483.

Maes, M., Vandewoude, M., Schotte, C., Maes, L., Martin, M., Sharpe, S. \& Blockx, P. (1990). The relationship between the cortisol response to dexamethasone and to L-5-HTP and the availability of L-tryptophan in depressed females. Biological Psychiatry 27, 601-608.

Moreno, F. L., Straver, G., Heninger, G. \& Delgado, P. (1995). Mood response to tryptophan and vulnerability to depression. Society for Neuroscience 21A (Abstract).

Neumeister, A., Praschak-Rieder, N., Hesselmann, B., Vitouch, O., Rauh, M., Barocka, A. \& Kasper, S. (1998). Effects of tryptophan depletion in fully remitted patients with seasonal affective disorder during the summer. Psychological Medicine 28, 257-264.

Nishizawa, S., Benkelfat, C., Young, S. N., Leyton, M., Mzengeza, S., de Montigny, C., Blier, P. \& Diksic, M. (1997). Differences between males and females in rates of serotonin synthesis in the human brain. Proceedings of the National Academy of Sciences 94, 5308-5313.

Ribeiro, S. C., Tandon, R., Grunhaus, L. \& Greden, J. F. (1993). The DST as a predictor of outcome in depression: a meta-analysis. American Journal of Psychiatry 150, 1618-1629.

Sapolsky, R. M., Krey, L. C. \& McEwen, B. (1986). The neuroendocrinology of stress and ageing: the glucocorticoid cascade hypothesis. Endocrinology Reviews 7, 284-301.

Smith, K. A., Clifford, E. M., Hockney, R. A., Clark, D. M. \& Cowen, P. J. (1997a). Effect of tryptophan depletion on mood in male and female volunteers: a pilot study. Human Psychopharmacology 12, 111-117.

Smith, K. A., Fairburn, C. G. \& Cowen, P. J. (1997b). Relapse of depression after rapid depletion of tryptophan. Lancet 349, 915-919.

Van Londen, L., Goekoop, J. G., Zwinderman, A. H., Lanser, J. B. K., Wiegant, V. N. \& De Wied, D. (1998). Neuropsychological performance and plasma cortisol, arginine vasopressin and oxytocin in patients with major depression. Psychological Medicine 28, 275-284.

Young, S. N., Smith, S. E., Phil, R. O. \& Ervin, F. R. (1985). Tryptophan depletion causes a rapid lowering of mood in normal males. Psychopharmacology 87, 173-177. 